\title{
Variability of in situ proteomic profiling and implications for study design in colorectal tumors
}

\author{
JIA-QING LI $^{1 *}$, BAOGANG J. XU ${ }^{2,7^{*}}$, BASHAR SHAKHTOUR ${ }^{6}$, NATASHA DEANE ${ }^{1}$, \\ NIPUN MERCHANT ${ }^{1,7}$, MARTIN J. HESLIN ${ }^{8}$, KAY WASHINGTON ${ }^{3,7}$, ROBERT J. COFFEY RA, $^{2,4,5,7}$, \\ R. DANIEL BEAUCHAMP ${ }^{1,2,5,7}$, YU SHYR ${ }^{6,7}$ and DEAN BILLHEIMER ${ }^{6,7}$ \\ Departments of ${ }^{1}$ Surgery, ${ }^{2}$ Cancer Biology, ${ }^{3}$ Pathology, ${ }^{4}$ Medicine, ${ }^{5}$ Cell and Developmental Biology, and ${ }^{6}$ Biostatistics , \\ Vanderbilt University Medical Center; ${ }^{7}$ Vanderbilt-Ingram Cancer Center, Nashville, TN 37232-2733; \\ ${ }^{8}$ Department of Surgery, University of Alabama at Birmingham, Birmingham, AL 35294-0016, USA
}

Received February 2, 2007; Accepted March 27, 2007

\begin{abstract}
Knowledge of intrinsic tumor heterogeneity is vital for understanding of tumor progression mechanisms as well as for providing efficient treatments. In situ proteomic profiling of tumors is a powerful technology with potential to enhance our understanding of tumor biology, but sources of variability due to patient and tumor heterogeneity are poorly understood and are the topic of this investigation. Clarification of variability within case and between cases is also important for designing future studies. Direct protein profiling by matrixassisted laser desorption/ionization time-of-flight mass spectrometry (MALDI-TOF MS) is a sensitive and powerful technology for obtaining hundreds of protein expression peaks from a thin tissue section. By combining robotic microspotting and laser capture microdissection with MALDI MS, we acquired multiple spectra per case to evaluate inter- and intra-case variability in human colorectal tumor and murine cecal carcinoma. We detected 256 peaks from 164 samples of 111 patients, which consisted of 55 normal colorectal mucosal samples, 24 adenomas, 71 primary carcinomas, and 14 hepatic metastases. In addition, we detected 291 peptide/protein peaks
\end{abstract}

Correspondence to: Dr Dean Billheimer, Biostatistics, 571 Preston Research Building, Vanderbilt-Ingram Cancer Center, Nashville, TN 37232-6848, USA

E-mail: dean.billheimer@vanderbilt.edu

${ }^{*}$ Contributed equally

Abbreviations: MALDI-TOF MS, matrix-assisted laser desorption/ ionization time-of-flight mass spectrometry; LCM, laser capture microdissection

Key words: in situ, proteomic profiling, colorectal tumor, variability from 34 orthotopically transplanted murine cecal carcinomas and 14 hepatic metastases. In human colorectal samples, we observed that proteomic profiling in adenomas was more homogeneous across patients than in normal mucosa specimens ( $\mathrm{p}=0.0008)$, but primary carcinoma exhibited greater heterogeneity than normal mucosa and adenomas (both $\mathrm{p}<0.0001$ ). Murine cecal carcinomas were homogeneous within and between carcinomas, while their hepatic metastases tended toward greater intra-tumor differences $(\mathrm{p}<0.0001)$. Inter- and intra-case variability was approximately equal for many protein peaks. Acquiring up to 5 subsamples per case could reduce the total number of cases required, but further reduction from additional subsampling was modest unless intra-case variability comprises a greater proportion of total variation (e.g. $>70 \%$ ). In summary, this study characterizes intra- and inter-case variability of high-throughput protein expression in colorectal tumors, and provides guidance for the sample numbers required for in situ proteomic studies.

\section{Introduction}

The molecular profiles within tumor tissues may vary substantially due to intrinsic tumor heterogeneity $(1,2)$. Increased knowledge of the complex molecular changes associated with tumor heterogeneity will improve our understanding of tumor biology, and may enable more accurate prognostication and individualized therapy. Additionally, determination of the tumor sample numbers in various study designs will also benefit from the quantitative comprehension of the overall protein expression dynamics within each tumor. Thus, systematic assessment of molecular profile variation both within each specimen and across a broad range of tumor types and grades is needed to better understand this heterogeneity. Numerous studies have explored different aspects of this question at the level of mRNA transcripts (1-4); however, quantitative and systematic analyses of tumor heterogeneity both within the same tumor and across different tumors for the in situ proteomic profiling have not been studied yet. To investigate the protein expression profile variability associated with tumor heterogeneity, an accurate and highthroughput proteomic technology is needed. 
Matrix-assisted laser desorption/ionization time-of-flight mass spectrometry (MALDI-TOF MS) has been used successfully to rapidly and concurrently profile the expression of hundreds of proteins directly from frozen tissue specimens (5-7). It has been used in mouse models of human cancer $(5,8-10)$, and in protein profiling of human specimens of normal and pathologic tissues (11-14). Advances in MALDI-TOF MS in situ sampling methods such as laser capture microdissection (LCM) (15) and robotic microspotting (16) allow histology-guided sampling of spatially heterogeneous tissue. By collecting multiple spectra from a selected histologic type from each tissue specimen, we can assess inter-case (e.g., patient, mouse) and intra-case variation. Inter-case variation describes the biologic heterogeneity between individuals in a population, while intra-case variation is associated with technical and biological factors such as instrument precision, matrix spot size, and tissue heterogeneity. Knowledge of these complementary components of variation can provide information about the sources of variability in protein profile, and allows comparisons of variability for different histologic types. In addition, such knowledge provides valuable information for planning future experiments and data collection to reduce the deleterious effects of nuisance heterogeneity.

In most studies adopting in situ proteomic profiling, micropipette was used for directly targeting tissue and applying matrix (5,7-14). Such targeting and application are simple, quick and cheap, but produce large (diameter $>2000$ microns), non-uniform spots that may cover a mixture of histologic features. An emerging technique, laser capture microdissection (LCM), has been introduced to more precisely target tiny area (diameter $<10$ microns) $(15)$. It provides the highest histologic specificity and purity, but consumes longer time to prepare sample. Another emerging technique, robotic microspotting, has recently been used to deposit matrix rapidly and automatically (17). This technique standardizes sample preparation, generates more uniform microspots (diameter $=200$ microns) with consistent ratios of tissue to matrix, and combines the high efficiency of applying matrix with high histologic specificity approaching that of LCM. Therefore, this study employs LCM to acquire mouse carcinomas, and uses robotic microspotting to target human colorectal tumors.

\section{Materials and methods}

Human specimen acquisition. As a part of the Gastrointestinal Cancer Special Program of Research Excellence (GI SPORE), Institutional Review Boards approved collecting specimens from Vanderbilt University, South Florida University, and University of Alabama at Birmingham. Following aquisition of patient consent, human tissue specimens were collected immediately after tumor resection, snap-frozen in liquid nitrogen, and then stored at $-80^{\circ} \mathrm{C}$ until analyzed. In total, 164 specimens from 111 patients with colorectal tumor were collected from the above listed institutions between 1999 and 2004. These tissues were grouped as normal mucosa $(n=55)$, adenoma (24), primary carcinoma $(n=71)$, and hepatic metastasis $(n=14)$. Normal mucosa was taken at least $10 \mathrm{~cm}$ away from the edge of primary carcinoma. Primary carcinomas, chosen consecutively, were evaluated in accordance with the tumor-node-metastasis classification of American Joint Committee on Cancer (AJCC) (18). All patients with primary or metastatic carcinoma were treated by curative procedure, and no patient received preoperative chemotherapy or radiotherapy.

Human specimen preparation. Twelve micrometer thick frozen sections from human colorectal samples were spread and thawed on gold plates, dried in vacuum (30 min), washed in ethanol (70\%: $30 \mathrm{sec} \times 2,100 \%: 15 \mathrm{sec})$ and dried in vacuum (5 min). Robotic microspotting (17) was used to apply $25 \mathrm{mg} / \mathrm{ml}$ sinapinic acid to targeted region under the conditions: start/ stop, 20 drops x 3; drop ejection rate, $10 \mathrm{~Hz}$. Spotted sections were dried in vacuum for $60 \mathrm{~min}(13,14,17)$.

Mouse carcinoma production. CT 26 cells were used to inoculate the cecae of syngeneic Balb/C mice under anesthesia with $0.2 \%$ isofluorane. To achieve this, a midline incision was made and the cecum brought out of the abdomen. Through a 25 gauge needle, $1 \times 10^{5}$ cells in $100 \mu \mathrm{l}$ of PBS buffer were injected into the seromuscular layer of the cecum on the antimesenteric border taking care not to inject intraluminally, and then the cecum was replaced into the abdomen and the incision closed with clips or suture. Mice reliably developed primary carcinoma within the cecum in 10 to 20 days and liver metastasis within 4 weeks at $60-70 \%$ penetrance. Tumors were harvested from the cecum and/or liver and half of the tumor was flash frozen in liquid nitrogen and stored at $-80^{\circ} \mathrm{C}$ while the remainder was fixed in $4 \%$ paraformaldehyde and then embedded by paraffin.

Mouse carcinoma preparation. Five micron thick frozen sections from mouse cecal carcinomas were dehydrated in ethanol (70\%: $30 \mathrm{sec}, 95 \%$ : $1 \mathrm{~min}, 100 \%$ : $1 \mathrm{~min} \times 2$ times), cleared in xylene (2.5 min x 2 times), and air dried. Carcinoma cells were precisely microdissected through the Arcturus PixCell II system (Mountain View, CA) using a laser-capture-microdissection phase microscope with a $30-\mu \mathrm{m}$ focus laser beam. After LCM, cells were captured onto thermoplastic film of a polymeric cap (Mountain View, CA) and mounted on a gold plate using a conductive double-sided tape (Digi-Key, Thief River Falls, MN). Then, sinapinic acid was manually microspotted on the captured cells under microscope through handpulled glass microcapillaries. Solvent was subsequently vaporized, allowing the matrix and proteins to co-crystallize $(15,19)$.

Mass spectrometry. Each spot received 150 randomly placed pulses with a $337 \mathrm{~nm}$ nitrogen laser in a Voyager Elite 2 MALDI-TOF mass spectrometer (PerSeptive Biosystems, Framingham, USA). Spectra were acquired using linear mode geometry under $25 \mathrm{kV}$ of accelerating potential and optimized delayed extraction conditions $(9,11,14,15)$.

Data processing. All spectra were processed using software developed in the Vanderbilt University High Dimensional Data Analysis Center (HDDAC). Human and mouse spectra were processed separately. Processing steps included baseline estimation and subtraction, calibration, total ion current 
normalization, peak selection, and binning of homologous peaks across spectra $(13,14,19)$. Once peak locations were identified by means of bin boundaries, signals were quantitated by computing the area under the spectrum between bin boundaries. The result was a computed area for each identified peak for all spectra. A logarithmic transform was applied to all peak areas to improve distributional characteristics. In our experience, the log peak areas exhibit unimodal distributions (approximately symmetric) that can be modeled using the Gaussian distribution.

Statistical analysis. A random effects linear model with random intercepts was used to model the log peak areas (20). A separate model was fit to each peak bin for each histologic type. This random effects model fits an exchangeable correlation structure to simultaneously estimate mean expression, inter-case, and intra-case components of variance. The exchangeable correlation structure presumes equal correlation between all observations (subsamples) from a single specimen. This common correlation is given by the intraclass correlation coefficient (ICC). ICC is defined by the expression:

$$
\mathrm{ICC}=\sigma_{\text {inter }}^{2} /\left(\sigma_{\text {inter }}^{2}+\sigma_{\text {intra }}^{2}\right)
$$

where $\sigma^{2}$ inter is the inter-case variance, and $\sigma^{2}$ intra is the intracase variance. As shown by equation i), ICC indicates the proportion of total variance attributable to inter-case heterogeneity. ICC is estimated by replacing variance components in equation i) with their estimates. The distributions across peaks for variance components and for ICC for each histologic type are summarized graphically using box-and-whisker plots (21).

To compare protein profile heterogeneity for two groups, we estimated the magnitude of variance components for each peak, and then summarized across all identified peaks in the spectrum. In more detail, we first counted the number of peaks for which the estimated variance component of histologic type I (e.g., primary carcinoma) exceeded the corresponding component of type II (e.g., normal mucosa). Under the null hypothesis of no difference in expression heterogeneity, one-half of the peaks will have the estimate for type I larger than that for type II, and one-half will have type I smaller than type II. The number of peaks counted can be compared with a binomial distribution with probability of success equal 0.5 , and sample size equal to the total number of protein peaks. Thus, we tested against the null hypothesis of a binomial distribution with equal probabilities. We used a two-sided test and a Bonferroni p-value adjustment $\left(\mathrm{p}_{\text {adjusted }}=10 \times \mathrm{p}_{\text {computed }}\right)$ to account for the five multiple comparisons conducted. In human, the comparisons were normal/adenoma, normal/carcinoma, adenoma/carcinoma, and carcinoma/metastasis; and in mice, the comparison is cecal tumor/hepatic metastasis. All the five comparisons were performed for inter-case variance and separately for intra-case variance. All parameter estimation, testing and other calculations were performed using the $\mathrm{R}$ statistics language (22).

Power and sample size analysis. Power and sample size calculations are based on a standard two-group comparison with Gaussian errors. In this setting, the standard sample size formula is given as follows:

$$
\mathrm{n}=2\left(\mathrm{z}_{1-\alpha / 2}+\mathrm{z}_{1-\beta}\right)^{2} / \delta^{2}
$$

where $\mathrm{n}$ is the number of cases required for each group, $\mathrm{z}$ denotes the quantile from a Gaussian distribution, $\alpha$ is the significance level (often 0.05 ), and $1-\beta$ is power. The effect size, $\delta$, is defined as follows:

$$
\delta=\left(\mu_{2}-\mu_{1}\right) / \sigma_{\text {total }}
$$

where $\mu_{1}$ and $\mu_{2}$ are the respective means for the two groups, and $\sigma_{\text {total }}$ is the common within group standard deviation. Each binned peak will have distinct values for $\mu_{1}, \mu_{2}$ and $\sigma_{\text {total }}$.

In studies with multiple observations per case (subsamples), within group variance $\left(\sigma_{\text {total }}^{2}\right)$ can be decomposed into two parts: inter-case (e.g., inter-patient, inter-mouse) variation and intra-case variation.

$$
\sigma_{\text {total }}^{2}=\sigma_{\text {inter }}^{2}+\sigma_{\text {intra }}^{2}
$$

If $m$ (independent) subsamples are acquired for each specimen, the effective total variance for each case will be reduced, and is described by:

$$
\sigma_{\text {total }}^{2}=\sigma_{\text {inter }}^{2}+\sigma_{\text {intra }}^{2} / m
$$

This expression for total variance can be combined with equation ii) to compute number of cases and number of subsamples required to achieve specified power and detection requirements. Note that by use of subsampling, the contribution of intra-case variability can be made negligibly small (in principle).

\section{Results}

Variation of spot histology in human colorectal tumors. Crystal spots formed by sinapinic acid were circular regions of approximately $200 \mu \mathrm{m}$ in diameter. Each spot typically occupied one to three crypts of colorectal glands as confirmed histologically (Fig. 1A-D). Crypts were lined by colonocytes and goblet cells, with rare endocrine cells, and were surrounded by a supporting stroma consisting of extracellular matrix, small vessels, lymphocytes and other inflammatory cells (Fig. 1E-H). In frozen sections, normal crypts exhibited a highly regular structure with epithelial cells lining the exterior of all crypt surfaces (Fig. 1E). Conversely, adenomas exhibited more structural variation including mucosal thickening with irregular branching of crypts (Fig. 1F). Primary and metastatic carcinoma frequently exhibited a more solid growth pattern, and presented a mixture involving stroma, and epithelial cells (Fig. 1G and H). Fig. 1E-H demonstrates how the histological features within a microspot varied with even slight shifting for position of a $0.2 \mathrm{~mm}$ microspot.

Result of data processing. Data processing of spectra from human colorectal normal mucosa, adenoma, primary carcinoma and hepatic metastasis resulted in detection of a total of 256 protein peaks in the 2,000-25,000 mass/charge $(\mathrm{m} / \mathrm{z})$ range. Example spectra from each of the tissue types are shown 


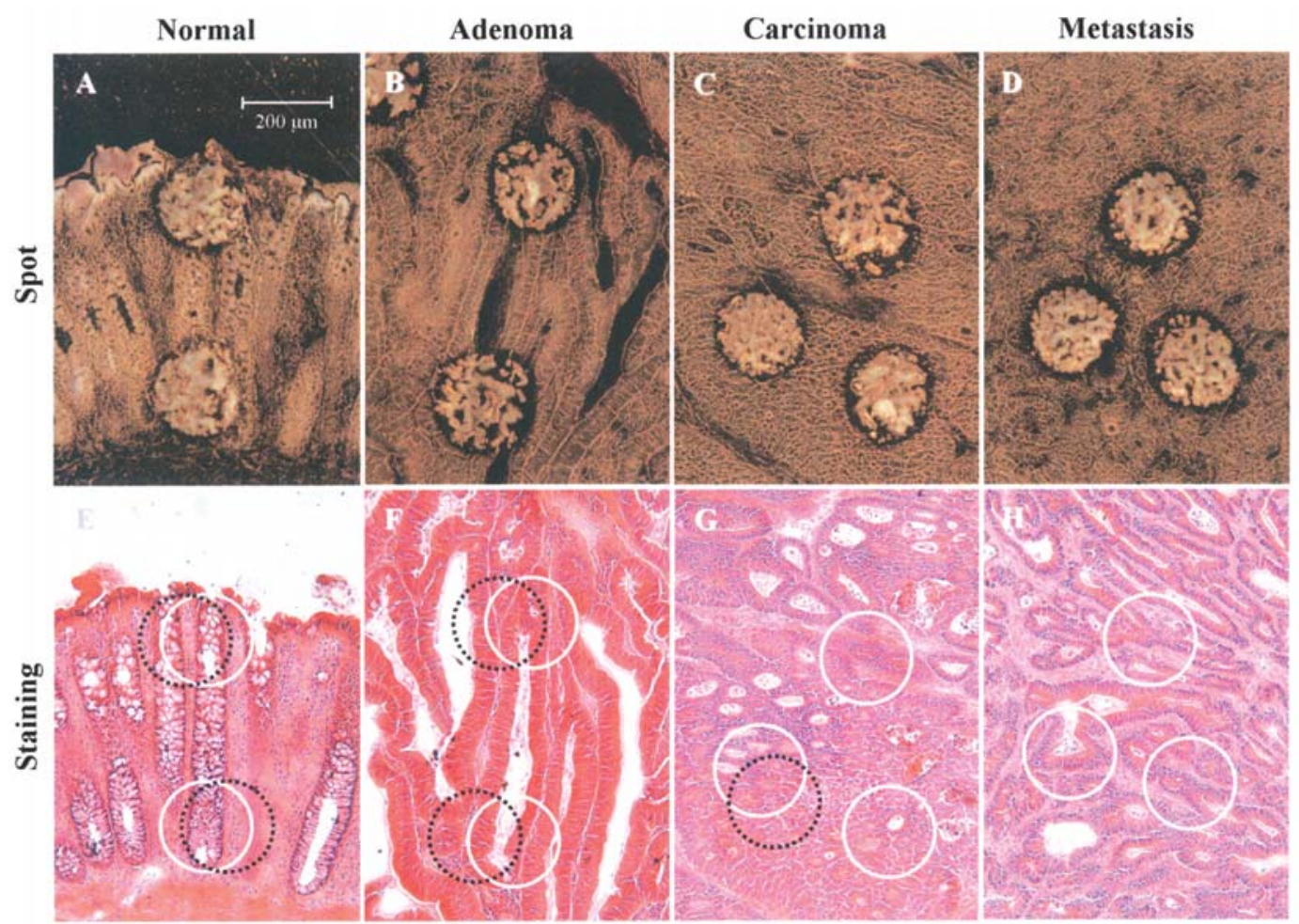

Figure 1. Variation of histologic features contained within microspots from human specimens. (A-D) Images of gold plate tissue sections indicate diameter, area and color of a crystal spot $\left(0.2 \mathrm{~mm}, 0.1256 \mathrm{~mm}^{2}\right.$, and bright yellow, respectively). Contours of histological structures are evident: cryptic cavity appears black; epithelial region appears bright yellow; stromal region appears deep yellow or shallow black. (E-H), Hematoxylin-eosin staining of serial tissue section confirms that the corresponding regions (white line circles) of crystal spots contain a mix of glandular cavity, cryptic epithelium and stroma. However, the ratio of the three components differs considerably between microspots at the top and the bottom of a normal crypt, between spots in adenoma, and among spots in primary carcinoma or hepatic metastasis. Histological features also vary as microspots are shifted slightly (white outline shifts to black dot outline).
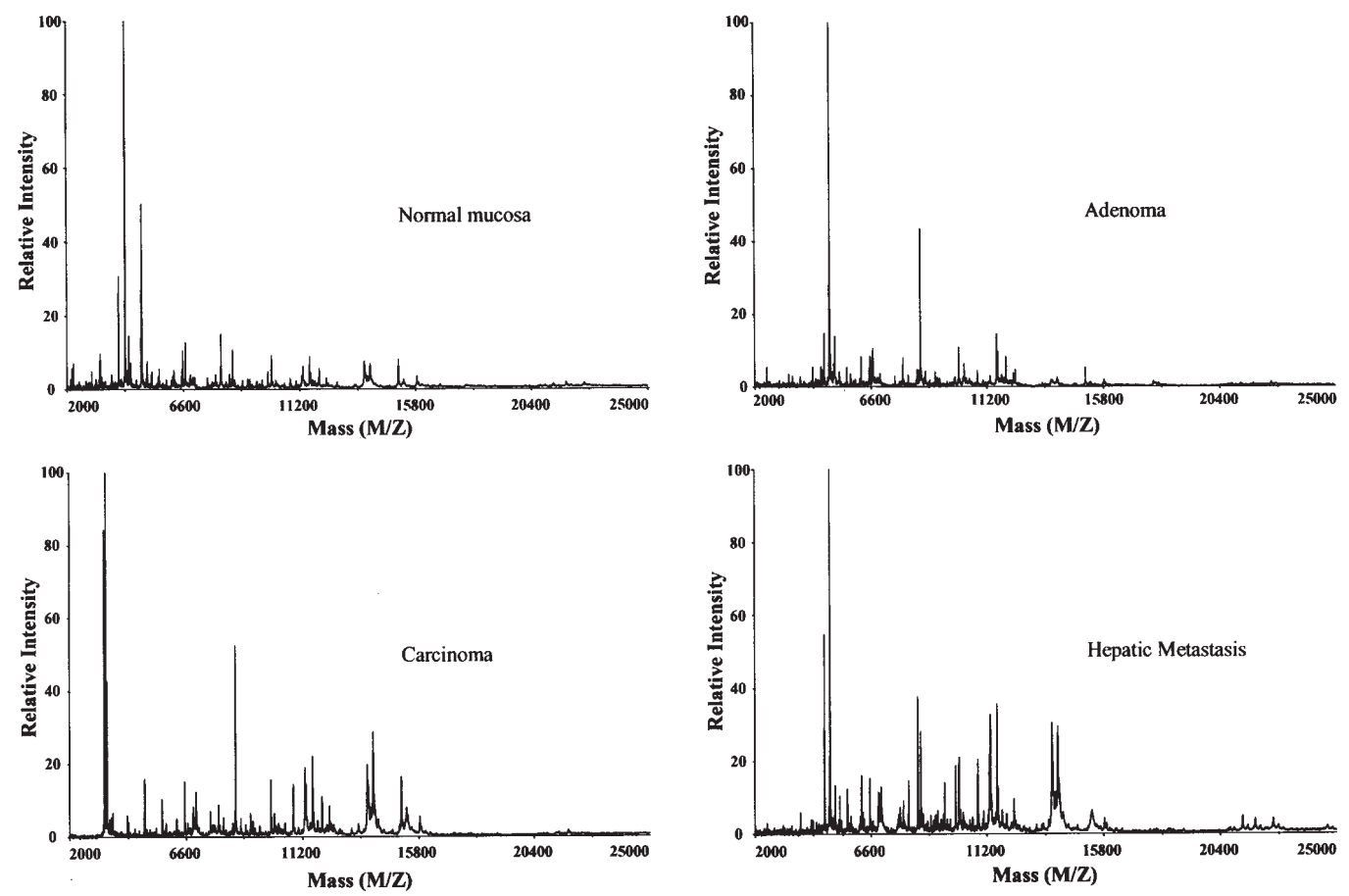

Figure 2. Protein profile spectra for human colorectal tissue. Normal mucosa, adenoma, primary carcinoma and hepatic metastasis exhibit 256 peaks in the range between 2,000 and 25,000 mass/charge as detected by MALDI-TOF MS.

in Fig. 2. Spectra from mouse cecal carcinomas and hepatic metastases produced a total of 291 peaks in the 2,000-25,000 mass/charge range. Example spectra from cecal carcinoma and hepatic metastasis are shown in Fig. 3. The number of 

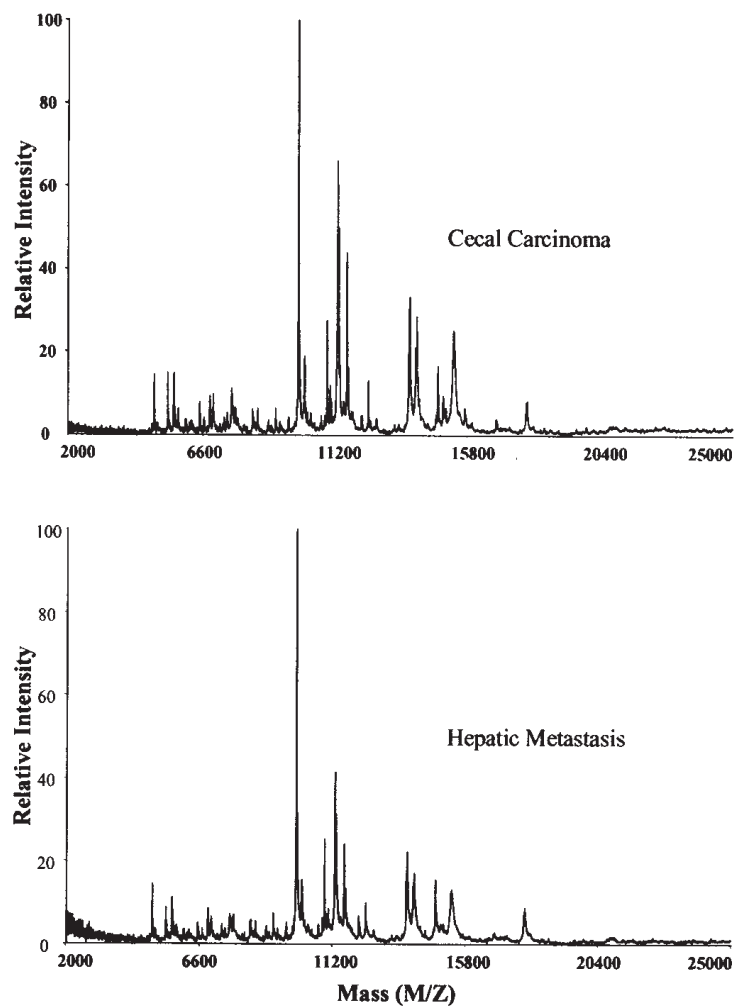

Figure 3. Protein profile spectra for mouse cecal carcinoma. Cecal tumors and hepatic metastases exhibit 291 peaks in the range between 2,000 and 25,000 mass/charge detected by MALDI-TOF MS.

Table I. Number of cases and spectra from colorectal tumors.

\begin{tabular}{lcc}
\hline Histologic type & Case no. & Spectral no. \\
\hline Human & & \\
Normal mucosa & 55 & 1045 \\
Adenoma & 24 & 399 \\
Primary carcinoma & 71 & 1944 \\
Hepatic metastasis & 14 & 309 \\
Mouse & & \\
Cecal carcinoma & 34 & 280 \\
Hepatic metastasis & 14 & 105 \\
\hline
\end{tabular}

spectra acquired from each histologic type is summarized in Table I.

Variability of inter- and intra-case in human and mouse tumor. The estimated standard deviations of inter- and intrapatient variability for eight selected peaks in human colorectal tissue are shown in Fig. 4. The eight peaks represent deduced
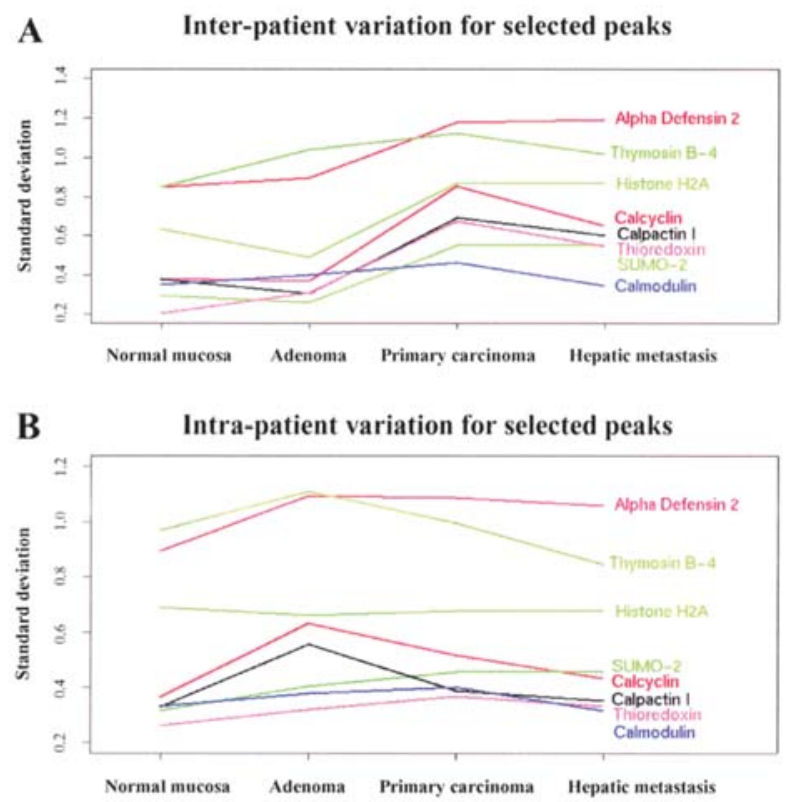

Figure 4. Standard deviations of selected peaks in human colorectal tissue. Estimated standard deviations of 8 selected peaks are shown for inter-patient (A) and intra-patient (B) variability in human colorectal tissue. The selected peaks are alpha-defensin 2 (m/z 3372), thymosin B-4 (4965), calcyclin (10091), SUMO-2 (10520), calpactin I (11072), thioredoxin (11652), histone H2A (14007), and calmodulin (16797) $(13,14,23-25)$.

A

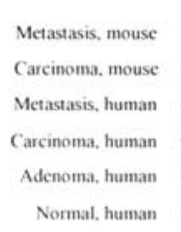

B

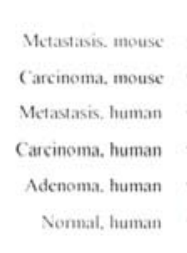

C

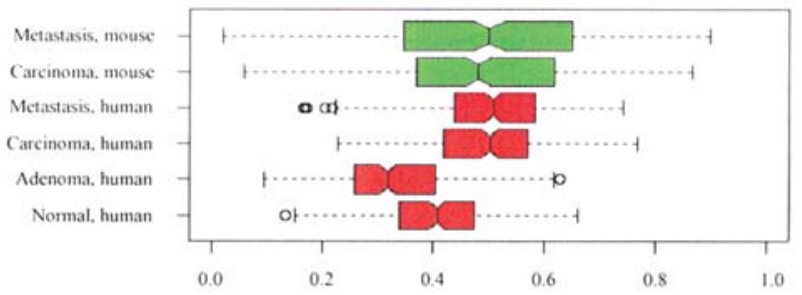

Figure 5. Distribution of inter-case and intra-case variability in colorectal tumors. Box-and-whisker plots summarize the distribution of estimated inter-case standard deviation (A), intra-case standard deviation (B), and intraclass correlation coefficient (C). Ends of colored boxes indicate the 25th and 75th percentiles of each distribution, respectively. The median (50th percentile) is indicated by a vertical line in each box. 'Whiskers' extend to observations up to $1.5 x$ interquartile range from the nearest box end. Notches associated with medians provide a visual guideline to the separation of medians. Specifically, if the notches from two box plots do not overlap, their medians would be judged different at approximately the 0.05 level (no adjustment for multiple comparisons). Red colored boxes indicate human colorectal tissue, and green boxes indicate murine carcinoma. 
Table II. Quantiles of inter-case standard deviation, intra-case standard deviation, and intraclass correlation coefficient in colorectal tumors.

Distribution quantile

\begin{tabular}{|c|c|c|c|c|c|}
\hline \multirow{2}{*}{$\begin{array}{l}\text { Index } \\
\text { Histologic type }\end{array}$} & \\
\hline & 0.05 & 0.25 & 0.5 & 0.75 & 0.95 \\
\hline \multicolumn{6}{|l|}{ Inter-case standard deviation } \\
\hline Normal mucosa, human & 0.23 & 0.31 & 0.39 & 0.49 & 0.65 \\
\hline Adenoma, human & 0.2 & 0.28 & 0.35 & 0.46 & 0.67 \\
\hline Primary carcinoma, human & 0.31 & 0.4 & 0.49 & 0.62 & 0.86 \\
\hline Hepatic metastasis, human & 0.28 & 0.37 & 0.46 & 0.58 & 0.84 \\
\hline Cecal carcinoma, mouse & 0.15 & 0.21 & 0.29 & 0.4 & 0.6 \\
\hline Hepatic metastasis, mouse & 0.14 & 0.21 & 0.29 & 0.41 & 0.6 \\
\hline \multicolumn{6}{|l|}{ Intra-case standard deviation } \\
\hline Normal mucosa, human & 0.3 & 0.39 & 0.48 & 0.59 & 0.78 \\
\hline Adenoma, human & 0.32 & 0.42 & 0.52 & 0.63 & 0.88 \\
\hline Primary carcinoma, human & 0.36 & 0.42 & 0.48 & 0.59 & 0.81 \\
\hline Hepatic metastasis, human & 0.32 & 0.38 & 0.45 & 0.56 & 0.76 \\
\hline Cecal carcinoma, mouse & 0.14 & 0.2 & 0.27 & 0.41 & 0.85 \\
\hline Hepatic metastasis, mouse & 0.14 & 0.2 & 0.3 & 0.43 & 0.92 \\
\hline \multicolumn{6}{|l|}{ Intraclass correlation coefficient } \\
\hline Normal mucosa, human & 0.25 & 0.34 & 0.41 & 0.47 & 0.59 \\
\hline Adenoma, human & 0.17 & 0.26 & 0.32 & 0.4 & 0.51 \\
\hline Primary carcinoma, human & 0.33 & 0.42 & 0.5 & 0.57 & 0.71 \\
\hline Hepatic metastasis, human & 0.28 & 0.44 & 0.51 & 0.58 & 0.67 \\
\hline Cecal carcinoma, mouse & 0.2 & 0.37 & 0.48 & 0.62 & 0.76 \\
\hline Hepatic metastasis, mouse & 0.12 & 0.34 & 0.5 & 0.65 & 0.78 \\
\hline
\end{tabular}

proteins that have been found at the corresponding $\mathrm{m} / \mathrm{z}$ values as described in previous studies $(13,14,23-25)$. Such protein peaks exhibited considerable variation amongst the different types of colorectal tissues. For instance, the estimated standard deviation of inter-patient variability for thymosin- $\beta 4$ increased from normal mucosa through adenoma to primary carcinoma, but decreased in hepatic metastasis. Fig. 5 shows the distribution of inter-case standard deviation, intra-case standard deviation and intraclass correlation co-efficient for all histologic tissue types that were analyzed. These distributions summarize all 256 detected peaks in human samples and 291 detected peaks in murine samples, and provide a global summary of protein variability for each histologic type. Summary statistics for these distributions are shown in Table II. In human normal mucosa, for example, selected quantiles $(5 \%, 25 \%, 50 \%, 75 \%$, $95 \%$ ) of inter-case standard deviation are $0.23,0.31,0.39$, 0.49 and 0.65 , respectively. In general, inter- and intra-case standard deviations were smaller in the murine cecal carcinomas than in human colorectal tissue.

A quantitative comparison of inter- and intra-case protein heterogeneity for different histologic types is summarized in Table III. This analysis produced a number of interesting results. One hundred and sixty (of $256,62 \%$ ) protein peaks in normal mucosa showed greater inter-patient variability than corresponding peaks in adenomas (adjusted p-value <0.0008). For $83 \%$ of all peaks, conversely, primary carcinomas exhibited greater heterogeneity between patients than normal mucosa (adjusted p-value $<0.0001$ ), and $87 \%$ of primary carcinoma peaks that exhibited a greater variability when compared with adenoma (adjusted p-value $<0.0001$ ). With regard to intra-patient variability, $69 \%$ of protein peaks in adenoma and $59 \%$ of peaks in primary carcinoma exhibited greater intra-patient heterogeneity than peaks from normal mucosa (adjusted p-value $<0.0001$ and $<0.05$, respectively). In addition, profiles from liver metastases appeared to be much more internally homogeneous than those from primary carcinomas (adjusted p-value $<0.0001$ ).

In mice, cecal carcinomas exhibited slightly less intermouse heterogeneity than the hepatic metastases, as $57 \%$ of protein peaks had larger inter-mouse variance estimates in metastatic tumors. However, after adjustment for multiple testing the p-value for this comparison was not significant at the 0.05 level (adjusted p-value $=0.19$ ). Similarly, intramouse variability of protein profiles was greater in hepatic metastases than in cecal carcinomas $(64 \%$, adjusted $p$-value $<0.0001)$.

Use of variance components in study design. Table IV combines the results of equations ii) and iv) to show requirements of case number ( $\mathrm{n}$, for example, non-bold numbers $6,4,4)$ for selected values of subsample (bold 1, 5, 20), intra-case variance (bold $0.2,0.5,1.0$ ), and inter-case variance (bold $0.2,0.5,1.0)$. Each calculation assumed a mean difference of 1 unit, $80 \%$ power, and a 0.05 significance level. For each 
Table III. Comparison for variability of proteomic profiling between colorectal tumors.

\begin{tabular}{|c|c|c|c|c|c|}
\hline Comparison & Percent of peaks $(\%)$ & $95 \% \mathrm{CI}^{\mathrm{a}}$ & p-value & $\mathrm{p}$-value, Adjusted & Larger variability \\
\hline \multicolumn{6}{|l|}{ Inter-patient standard deviation } \\
\hline Normal vs. adenoma & 62 & $32-44$ & $<0.0001$ & 0.0008 & Normal \\
\hline Normal vs. carcinoma & 83 & $78-88$ & $<0.0001$ & $<0.0001$ & Carcinoma \\
\hline Adenoma vs. carcinoma & 87 & $82-91$ & $<0.0001$ & $<0.0001$ & Carcinoma \\
\hline Carcinoma vs. metastasis & 43 & $36-49$ & 0.02 & 0.2 & Not significant \\
\hline \multicolumn{6}{|l|}{ Intra-patient standard deviation } \\
\hline Normal vs. adenoma & 69 & $63-74$ & $<0.0001$ & $<0.0001$ & Adenoma \\
\hline Normal vs. carcinoma & 59 & $53-65$ & 0.005 & 0.05 & Carcinoma \\
\hline Adenoma vs. carcinoma & 44 & $38-50$ & 0.05 & 0.5 & Not significant \\
\hline Carcinoma vs. metastasis & 21 & $16-26$ & $<0.0001$ & $<0.0001$ & Carcinoma \\
\hline \multicolumn{6}{|l|}{ Inter-mouse standard deviation } \\
\hline Carcinoma vs. metastasis & 57 & $51-63$ & 0.02 & 0.19 & Not significant \\
\hline \multicolumn{6}{|l|}{ Intra-mouse standard deviation } \\
\hline Carcinoma vs. metastasis & 64 & $58-69$ & $<0.0001$ & $<0.0001$ & Metastasis \\
\hline
\end{tabular}

${ }^{\mathrm{a} C}$ Confidence interval.

Table IV. Requirements of case number for selected values of subsample, intra-case variance, and inter-case variance.

\begin{tabular}{|c|c|c|c|c|c|c|c|c|c|}
\hline \multirow{4}{*}{$\begin{array}{l}\text { Subsample } \\
\text { No. }(\mathrm{m})\end{array}$} & \multicolumn{9}{|c|}{ Intra-case variance } \\
\hline & \multicolumn{3}{|c|}{0.2} & \multicolumn{3}{|c|}{0.5} & \multicolumn{3}{|c|}{1.0} \\
\hline & \multicolumn{3}{|c|}{ Inter-case variance } & \multicolumn{3}{|c|}{ Inter-case variance } & \multicolumn{3}{|c|}{ Inter-case variance } \\
\hline & 0.2 & 0.5 & 1.0 & 0.2 & 0.5 & 1.0 & 0.2 & 0.5 & 1.0 \\
\hline 1 & 6 & 11 & 19 & 11 & 16 & 24 & 19 & 24 & 32 \\
\hline 5 & 4 & 9 & 17 & 5 & 10 & 17 & 7 & 11 & 19 \\
\hline 20 & 4 & 8 & 16 & 4 & 9 & 16 & 4 & 9 & 17 \\
\hline
\end{tabular}

Assumptions: mean difference, $\mu_{2}-\mu_{1}=1$; Power: $80 \%, \alpha=0.05$ (two-sided).

pair of variance component values, there were multiple combinations of case number (n) and subsample number (m) that could satisfy requirements of the power and significance level. For example, when inter-case variance and intra-case variance each equaled 0.5 , a one unit shift in mean could be detected using: i) $n=16$ and $m=1$; ii) $n=10$ and $m=5$; or iii) $\mathrm{n}=9$ and $\mathrm{m}=20$; all with $80 \%$ power, 0.05 significance level. With this type of information regarding the magnitude of variance components, we may design experimental sampling plans to control the effects of inter-case and intra-case variation.

\section{Discussion}

In this study, we described the inter- and intra-case variability in multistage human colorectal tumors and murine cecal carcinomas and hepatic metastasis. Protein profiles obtained from 3697 microspots of 111 colorectal cancer patients and
385 microspots from 34 mouse samples were statistically analyzed. By comparing variance estimates from different histological types we gained insight into protein expression heterogeneity for different tumor stages. Further, we described an approach to select appropriate sampling designs - specifying both the number of patients/mice, as well as the number of sampling locations (subsamples or 'spots') within a tissue section for in situ profiling experiments.

To quantitatively analyze the tumor heterogeneity, we defined our study to investigate the inter-case and intra-case protein expression variability. Inter-case variation reflects the biological variation between individuals within the same experimental group. Its effect on statistical inference is controlled by the number of cases selected for each group. Intra-case variation reflects instrument precision, tissue heterogeneity, and other factors contributing to measurement mismatch within a specimen. For in situ profiling studies, spatial heterogeneity of histology contributes to this component of variance. Intra- 
case variation is controlled by acquiring multiple replicate spectra from each specimen. These subsamples can be combined in data analysis to provide a more stable estimate of each specimen's profile response. Information regarding the magnitude of interand intra-case variance allows efficient experiment planning by balancing these complementary sources of variability. We note that this study focuses on the variability of protein expression, rather than mean levels of expression. Results of mean expression comparisons will be reported elsewhere.

Many tissues exhibit highly organized spatial structure that may be heterogeneous at a fine scale. The histologic features at one location might vary considerably from those at a neighboring location within the same specimen (26). Thus, single sample spots in isolation may not reliably represent the molecular signature of a given specimen, and multiple subsamples are required. In addition to more reliable estimates of mean response for a tissue specimen, multiple subsamples allow estimation of inter- and intra-specimen heterogeneity of protein expression profiles. Histologically guided sampling allows interrogation of specific histologic features, and spatial averaging of protein profiles when multiple subsamples are collected for each specimen $(15,17)$. Spatial averaging results in more stable estimates of protein content, and mitigates the effects of measurement error. Further, subsampling provides information regarding intra-specimen variability, as well as inter-specimen heterogeneity. Such knowledge of 'between' and 'within' variances provides insight into the stability of protein expression profiles.

When comparing protein profiles across patients, we observe that adenomas are more homogeneous across patients than are normal mucosa specimens. This suggests that adenomas may arise from a common mechanism (or a few mechanisms) exhibiting relatively tight control of protein expression. This may be consistent with the frequent inactivating mutation of the $A p c$ gene as the initiating event in the majority of colorectal adenomas/carcinomas (27). In contrast, primary carcinoma and hepatic metastases exhibit greater heterogeneity across patients than do normal mucosal samples. Such heterogeneity in protein profiles demonstrates multiple phenotypes of colorectal cancer and metastasis. When protein profiles from primary tumors are compared with those from metastatic tumors, the data provide a weak indication of reduced heterogeneity across multiple patients' metastases. When primary carcinomas are derived from a common cell type, as in the murine cecal carcinoma model, hepatic metastases tend toward greater intermouse differences than the orthotopically grafted cecal tumors. Although this result superficially contradicts the result in human carcinomas, it may be explained by the homogeneous starting material provided by the CT-26 cell inoculation.

With regard to intra-specimen variability, adenomas and primary carcinoma exhibit greater intra-tumor heterogeneity of protein profiles than normal mucosa. This result may be attributable to greater structural heterogeneity of tumors. Protein profiles for hepatic metastases are more homogeneous (internally) than those for primary tumors. The results for mouse tumors indicate that profiles for cecal carcinomas are more homogeneous than for hepatic metastases. This is likely a result of the structural homogeneity of the cecal carcinomas that were all derived from the same cultured cell line (CT-26).
When intra-case variability contributes substantially to total variation, a reduction in sample size (e.g., number of cases) can be achieved by subsampling. In primary carcinomas, the median ICC is 0.5 , indicating that one-half of the total variation is attributable to inter-patient sources and one-half to intra-patient sources. For an ICC of 0.5 , our results indicate substantial reductions in number of cases by increasing the subsampling from 1 to 5 spectra per case. Conversely, further increasing to 20 spectra per case results in little further reduction in variability. In settings where the intra-case variability is large and ICC is small, such as the case we observe with adenomas, further gains can be realized by additional subsampling.

Efficient experimental design relies on balancing the complementary components of variance. In situations in which the cost of case acquisition is expensive and the cost of incremental subsampling cheap (e.g., microspotting), then within group variance can be reduced by increasing the number of subsamples. Alternatively, if the cost of acquiring additional subsamples is time-consuming or expensive, then more cases may be required to achieve adequate statistical power. If estimates of inter- and intra-case variance are available (e.g. from preliminary data), the usual sample size calculations can be easily adapted to accommodate subsampling. Our results demonstrate that different resource allocations to the number of cases and the number of subsamples can achieve the same statistical power.

In conclusion, we have characterized inter- and intraspecimen variance for MALDI-TOF MS protein peaks in a collection of tissue specimens from human multistage colorectal tumors and murine cecal carcinomas. In addition, we have shown that information regarding 'between' and 'within' variances is useful for study planning. Specifically, additional subsamples within a specimen can, to a limited extent, be substituted for additional cases.

\section{Acknowledgments}

The authors thank Richard M. Caprioli, Hans-Rudolf Aerni, Pierre Chaurand and Dale S. Cornett of the Mass Spectrometry Research Center for providing valuable technical support during the experiment, thank Prashant R. Joshi of Department of Surgery for collecting colorectal samples, and thank Shuo Chen of Department of Biostatistics for assistance in performing statistical calculations. The authors acknowledge financial support from the National Institute of Health: P50CA95103, CA46413 and UO1CA84239 to R.J.C. and CA69457 and DK52334 to R.D.B. and from the Digestive Disease Center support grant: DK58404, and from the Vanderbilt-Ingram Cancer Center support grant: CA68485.

\section{References}

1. Blackhall FH, Pintilie M, Wigle DA, Jurisica I, Liu N, Radulovich N, Johnston MR, Keshavjee S and Tsao MS: Stability and heterogeneity of expression profiles in lung cancer specimens harvested following surgical resection. Neoplasia 6: 761-767, 2004.

2. Francis P, Fernebro J, Eden P, Laurell A, Rydholm A, Domanski HA, Breslin T, Hegardt C, Borg A and Nilbert M: Intratumor versus intertumor heterogeneity in gene expression profiles of soft-tissue sarcomas. Genes Chromosomes Cancer 43: 302-308, 2005.

3. O'Sullivan M, Budhraja V, Sadovsky Y and Pfeifer JD: Tumor heterogeneity affects the precision of microarray analysis. Diagn Mol Pathol 14: 65-71, 2005. 
4. Jacobsen M, Repsilber D, Gutschmidt A, Neher A, Feldmann K, Mollenkopf HJ, Kaufmann SH and Ziegler A: Deconfounding microarray analysis-independent measurements of cell type proportions used in a regression model to resolve tissue heterogeneity bias. Methods Inf Med 45: 557-563, 2006.

5. Chaurand P, Sanders ME, Jensen RA and Caprioli RM: Proteomics in diagnostic pathology: profiling and imaging proteins directly in tissue sections. Am J Pathol 165: 1057-1068, 2004.

6. Caldwell RL and Caprioli RM: Tissue profiling by mass spectrometry: a review of methodology and applications. Mol Cell Proteomics 4: 394-401, 2005.

7. Reyzer ML and Caprioli RM: MALDI mass spectrometry for direct tissue analysis: a new tool for biomarker discovery. J Proteome Res 4: 1138-1142, 2005.

8. Chaurand P, Da Gue BB, Ma S, Kasper S and Caprioli RM: Strain-based sequence variations and structure analysis of murine prostate specific spermine binding protein using mass spectrometry. Biochemistry 40: 9725-9733, 2001.

9. Reyzer ML, Caldwell RL, Dugger TC, Forbes JT, Ritter CA, Guix M, Arteaga CL and Caprioli RM: Early changes in protein expression detected by mass spectrometry predict tumor response to molecular therapeutics. Cancer Res 64: 9093-9100, 2004.

10. Xie L, Xu BJ, Gorska AE, Shyr Y, Schwartz SA, Cheng N, Levy S, Bierie B, Caprioli RM and Moses HL: Genomic and proteomic analysis of mammary tumors arising in transgenic mice. J Proteome Res 4: 2088-2098, 2005.

11. Schwartz SA, Weil RJ, Johnson MD, Toms SA and Caprioli RM: Protein profiling in brain tumors using mass spectrometry: feasibility of a new technique for the analysis of protein expression. Clin Cancer Res 10: 981-987, 2004

12. Rahman SM, Shyr Y, Yildiz PB, Gonzalez AL, Li H, Zhang X, Chaurand P, Yanagisawa K, Slovis BS, Miller RF, Ninan M, Miller YE, Franklin WA, Caprioli RM, Carbone DP and Massion PP: Proteomic patterns of preinvasive bronchial lesions. Am J Respir Crit Care Med 172: 1556-1562, 2005.

13. Schwartz SA, Weil RJ, Thompson RC, Shyr Y, Moore JH, Toms SA, Johnson MD and Caprioli RM: Proteomic-based prognosis of brain tumor patients using direct-tissue matrixassisted laser desorption ionization mass spectrometry. Cancer Res 65: 7674-7681, 2005.

14. Yanagisawa K, Shyr Y, Xu BJ, Massion PP, Larsen PH, White BC, Roberts JR, Edgerton M, Gonzalez A, Nadaf S, Moore JH, Caprioli RM and Carbone DP: Proteomic patterns of tumour subsets in non-small cell lung cancer. Lancet 362: 433-439, 2003.

15. Xu BJ, Caprioli RM, Sanders ME and Jensen RA: Direct analysis of laser capture microdissected cells by MALDI mass spectrometry. J Am Soc Mass Spectrom 13: 1292-1297, 2002.
16. Cornett DS, Mobley JA, Dias EC, Andersson M, Arteaga CL, Sanders ME and Caprioli RM: A novel histology-directed strategy for MALDI-MS tissue profiling that improves throughput and cellular specificity in human breast cancer. Mol Cell Proteomics 5: 1975-1983, 2006.

17. Aerni HR, Cornett DS and Caprioli RM: Automated acoustic matrix deposition for MALDI sample preparation. Anal Chem 78: 827-834, 2006.

18. Greene FL, Page DL, Fleming ID, Fritz A, Balch CM, Haller DG and Morrow M: AJCC Cancer Staging Manual. 6th edition. Springer, New York, 2002.

19. Xu BJ, Shyr Y, Liang X, Ma LJ, Donnert EM, Roberts JD, Zhang X, Kon V, Brown NJ, Caprioli RM and Fogo AB: Proteomic patterns and prediction of glomerulosclerosis and its mechanisms. J Am Soc Nephrol 16: 2967-2975, 2005.

20. Neter J, Kutner MH, Nachtsheim CJ and Wasserman W: Applied Linear Statistical Models. 4th edition. Irwin Publishing, Chicago, IL, 1996.

21. Tukey JW: Exploratory Data Analysis. Addison-Wesley, Reading, 1977.

22. R-Development-Core-Team: R: A Language and Environment for Statistical Computing. R Foundation for Statistical Computing, Vienna, 2006.

23. Masumori N, Thomas TZ, Chaurand P, Case T, Paul M, Kasper S, Caprioli RM, Tsukamoto T, Shappell SB and Matusik RJ: A probasin-large $\mathrm{T}$ antigen transgenic mouse line develops prostate adenocarcinoma and neuroendocrine carcinoma with metastatic potential. Cancer Res 61: 2239-2249, 2001.

24. Pierson J, Norris JL, Aerni HR, Svenningsson P, Caprioli RM and Andren PE: Molecular profiling of experimental Parkinson's disease: direct analysis of peptides and proteins on brain tissue sections by MALDI mass spectrometry. J Proteome Res 3: 289-295, 2004.

25. Roesch-Ely M, Nees M, Karsai S, Ruess A, Bogumil R, Warnken U, Schnolzer M, Dietz A, Plinkert PK, Hofele C and Bosch FX: Proteomic analysis reveals successive aberrations in protein expression from healthy mucosa to invasive head and neck cancer. Oncogene 26: 54-64, 2007.

26. Fletcher CDM: Diagnostic Histopathology of Tumors. Vol. 1, 2nd edition. Harcourt Publishers Ltd., London, pp171-930, 2000 .

27. Fearon ER and Vogelstein B: A genetic model for colorectal tumorigenesis. Cell 61: 759-767, 1990. 